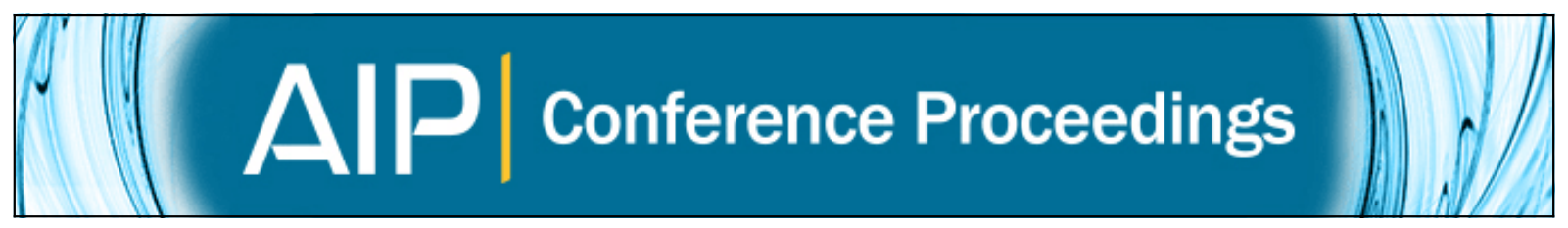

Otoacoustic Emissions (Part I) and central auditory effects: A moderated discussion

Wei Dong and Sarah Verhulst

Citation: AIP Conference Proceedings 1703, 090010 (2015); doi: 10.1063/1.4939408

View online: http://dx.doi.org/10.1063/1.4939408

View Table of Contents: http://scitation.aip.org/content/aip/proceeding/aipcp/1703?ver=pdfcov

Published by the AIP Publishing

Articles you may be interested in

Otoacoustic Emissions (Part II): A moderated discussion

AIP Conf. Proc. 1703, 090014 (2015); 10.1063/1.4939412

Cochlear Micromechanics (Part II): A moderated discussion

AIP Conf. Proc. 1703, 070010 (2015); 10.1063/1.4939384

Cochlear Micromechanics (Part I): A moderated discussion

AIP Conf. Proc. 1703, 070006 (2015); 10.1063/1.4939380

Molecular and Cellular Mechanics (Part I): A moderated discussion

AIP Conf. Proc. 1703, 030007 (2015); 10.1063/1.4939322

Ear-canal acoustic admittance and reflectance effects in human neonates. I. Predictions of otoacoustic emission and auditory brainstem responses

J. Acoust. Soc. Am. 113, 389 (2003); 10.1121/1.1523387 


\title{
Otoacoustic Emissions (Part I) and Central Auditory Effects: A Moderated Discussion
}

\author{
Wei Dong ${ }^{*, \dagger, *}$ and Sarah Verhulst ${ }^{* *, *}$ \\ *Research Service, VA Loma Linda Healthcare System, Loma Linda, California, USA \\ ${ }^{\dagger}$ Department of Otolaryngology, Head \& Neck Surgery, Loma Linda University, Loma Linda, California, USA \\ ${ }^{* *}$ Cluster of Excellence Hearing4All and Medizinische Physik, Department of Medical Physics and Acoustics, \\ University of Oldenburg, Oldenburg, Germany \\ *These authors contributed equally to this work
}

\begin{abstract}
The following is an edited transcript of a recorded discussion session on the topics of "Otoacoustic Emissions" and "Central Auditory Effects". The discussion, moderated by the authors, took place at the $12^{\text {th }}$ International Workshop on the Mechanics of Hearing held at Cape Sounio, Greece, in June 2014. All participants knew that the session was being recorded. In view of both the spontaneous nature of the discussion and the editing, however, this transcript may not represent the considered or final views of the participants, and may not represent a consensus of experts in the field. The reader is advised to consult additional independent publications.
\end{abstract}

\section{DISCUSSION SESSION}

Sarah Verhulst: I will put up a slide with some of the main topics we addressed in the talks and in the posters. It is important to consider the applications of otoacoustic emissions, because we've learned a lot about their generation mechanisms, and we have evolved from a yes/no screening answer to more precise measures of individual hearing status. We could discuss the challenges and the strengths of these methods and then move on to other topics. So is there anyone who wants to take a stab at this?

\section{Coherent Reflection and Cochlear Irregularities}

Bastian Epp: May I first ask a different question? Regarding the coherent reflection mechanism, if I look at the integral and the physical mechanism, a reflection occurs, and if the spatial frequency matches the wavelength, you get coherent reflection. But in many talks and papers, they mention that the reflection only comes from the peak region [of the traveling wave]. Looking at the integral, I think it mainly comes from the peak region, but it can also come from the tails. Chris [Shera], can you say something to that? I'm not very surprised by SFOAEs coming from more basal regions. I think that's totally consistent.

Wei Dong: I have a quick comment on the generation of the reflection components. As I showed in intracochlear responses, the $2 \mathrm{f} 2 \mathrm{f} 1$ actually originates from a broader region, at least a half octave away from $\mathrm{f} 2$ peak. The peak region contributes predominantly, but the generation site is not limited to the peak region only.

Christopher Shera: What the coherent reflection model says is that in principle, any irregularity anywhere that's excited by the traveling wave contributes reflected wavelets. But the reflected wavelets are really only coherent, as you said, when the spatial frequency matches the wavelength. The amplitude of the emission is determined both by that and by the amplitude of the forward traveling wave. And so, although the model says that in principle, emissions come from all cochlear regions stimulated by the traveling wave, it also says that whenever the peak of the traveling wave is sort of tall and broad, they tend to come predominantly from the peak region. So, anyone who says they come only from the peak region is sort of mischaracterizing it.

Carolina Abdala: So the irregularity issue and where it comes from and where it's coherent, and where we can surmise that the ear canal measurement arises from (or is coherent enough to get a strong enough response from), is of interest because I think this irregularity issue is kind of confusing me, too. I'd like to have clarification, that the irregularity isn't necessarily an anatomical thing. It could be. But it's not something that you can always see with our available techniques. So, I think there's some misconception that we have to see it, or else it's not there and might not be producing reflection.

Christopher Shera: Yeah, I agree with that. I guess the bottom line is that at the moment we don't really know what the dominant irregularity that is making the emissions is. Neither do we know if there is such a thing that one could identify as being the same across people. It could be that different people have different answers to that question. One question that was raised earlier had to do with whether the irregularities are dynamic. Some evidence was presented today by John Guinan that maybe 
they can be induced by efferent effects. They may also vary to some extent with stimulus intensity. One thing that we do know is that whatever the irregularities are at a given stimulus level, they are sufficiently stable that the emissions themselves are sort of stable over time. So if I measure your emissions today, I'll get the same answer if I measure them tomorrow (assuming that you haven't gone to a concert in the meantime), a month or a couple of years from now. So they're pretty stable over time, but that doesn't mean they don't vary with efferent stimulation or with different levels.

Glenis Long: I wanted to add that these are not monstrous irregularities that we're imagining in the model. These are very small, nearly random fluctuations. I think a lot of people have images of these being large things we should be able to see. And that's not the way the models are working.

Jonathan Siegel: Part of my concern about this is that as an experimentalist I like to measure things. It almost seems like we're treading into the realm of religion to postulate that there must exist this unknowable, unseeable, unmeasurable irregularity. If you try to flip this whole story around, is it not possible that the same pattern of emissions that we measure couldn't be generated by an array of hair cell force generators that are nonlinear, but also have some irregularity for some reason. I think David Kemp had suggested from the very beginning that there must be some kind of irregularity in the cochlea, otherwise we wouldn't see the long delay of these so called place fixed emissions. Are we really talking about something different? I guess that bothers me.

Dáibhid Ó Maoiléidigh: Chris Shera should feel free to completely correct me on this. My impression is that in both mechanisms, whether you talk about coherent reflection, or about little oscillators sitting on the membrane generating waves, in both cases you need something sitting on the membrane generating energy. The question is: does the energy simply propagate to the eardrum interface and immediately leave? Or is some of that energy reflected off that interface and come back and over several cycles build up? So then you just need to modulate the reflectance at the ear drum and you could get either case. Now, the irregularities themselves could be an independent phenomenon from the little oscillators or little local sources of activity, or they could be intrinsically linked. Or, you could have combination of the two. It seems difficult to me at the moment to distinguish these scenarios, but the two views are not completely divergent from each other.

Christopher Shera: It sounds to me, Dáibhid [Ó Maoiléidigh], that you're talking about the generation of spontaneous otoacoustic emissions, and my comments were addressed to the generation of stimulus frequency emissions and distortion products. I think the generation of spontaneous emissions is related to the mechanisms that you talked about. As to John Siegel's point, I don't regard the irregularities necessarily as being unmeasurable. I think if you look in the anatomy, you do see irregularity. We just don't know whether the irregularity we see is what's responsible for stimulus frequency emissions. Given that we're dealing with biological systems here, I think it would be far more a matter of religion to say that the mechanical characteristics varied smoothly rather than irregularly. But I certainly agree that irregularity and nonlinearity, they're both kind of intrinsic features of the cochlea. One of those features, the irregularity, can produce long latency emissions, just by itself, without any nonlinear mechanisms. The other category, the nonlinearity, can create short latency distortion product emissions without any contribution from irregularity. So together the two can produce what we observe.

Amin Saremi: First of all I want to thank John Guinan for the very informative presentation, and more important, the hypothesis that he brought up. So the take home message was that when you activate the MOC network, not all fibers are elicited. That causes irregularities that increase the emissions. So how is the distribution of these MOC-induced irregularities along the cochlear duct? Is it more in basal region or in apical region?

John Guinan: The innervation from the medial efferents peaks in the upper basal turn in most mammals, and the contralateral peaks a little more basal than the ipsilateral. So there's a pretty well-known distribution. If you stimulate the efferents in the floor of the fourth ventricle, where you may not stimulate them all, but you have a pretty equal probability, there's no tonotopic organization there, you get a pattern of inhibition in single auditory nerve fibers, or if you look at compound action potentials, that mimics approximately the overall innervation density. Fibers in all regions, in the apex and the base, are irregular, and I guess there is some difference in how irregular they are. Chris Brown has a paper that's just about to come out in General Comparative Neurology that compares labeled fibers along the apex to base. They're a little bit more spread out in the apex than they are in the base. That, I suppose, is consistent with the fact that the tuning is more spread out.

Renata Sisto: Just a comment for John Siegel about irregularity. You said that the irregularities look like God, because they are not measurable. But I think they are, because they are just a gradient in the cochlear impedance. If you had not this small gradient in the cochlear impedance, it would not be possible to have a reflection backward, because there is a theorem. [Without irregularities,] You would have a complete absorption of the energy at the best frequency place. So you have to think about a gradient in cochlear impedance, and you can also measure where this impedance gradient should be located, because you can identify the position of the maximum reflection by measuring the SFOAE spectrum, and identify where the energy spots are located. So you must think about a mechanism that makes the impedance not perfectly smooth. This mechanism can be whatever you want, from the physiological point of view.

Jonathan Siegel: Yes, I agree, I'm not doubting that this must exist. I'm just worried about that in the chinchilla, I can't look inside see what's causing the irregularity. I would be interested in hearing what David Kemp has to say about all this. He's sitting very quietly. [LAUGHTER] 
David Kemp: Well, I thought Mary Ann's [Cheatham] presentation was really revolutionary. She's changed a mouse into a human in the most important respect. [LAUGHTER] I thought that's tangible evidence of what might be causing irregularities. We haven't seen anything histologically, in humans anyway, and so maybe that's the first clue, that we should look at the tectorial membrane more than the hair cells.

Tobias Reichenbach: I wanted to follow up on John Siegel's comments, because that's also something that I've already asked. Although coherent reflection is such a beautiful theory, it seems hard to gain more experimental evidence. That's why we looked at alternative mechanisms for creating this long latency component. We've done theoretical and experimental work that shows that waves on Reissner's membrane can indeed constitute an alternative mechanism. That does not mean that one or the other has to be right. There could be a combination of reflections of different waves traveling in different modes. I wonder if the better way forward is to look at those alternative modes of propagation, based on Reissner's membrane, tectorial membrane, or on fast pressure waves that Heindrik [Duifhuis] has proposed and see if those suffice to explain the experimental results. I would be interested to hear what people think.

Geoffrey Manley: Two points. One is that in 1984 I proposed an idea for the rather regular distribution of spontaneous emissions in mammals based upon the coiling of the mammalian cochlea and the anatomical constraints that it imposes. At that time we were concentrating on localized sources of emissions, which could also be viewed as locations for impedance jumps. But in anticipation of this afternoon, if you're interested in the places where SFOAE are strong, or where spontaneous emissions occur, come to the talk which I will give on behalf of Christine Köppl and Chris Bergevin, on our recent work on barn owls. The bird cochlea has a much less regular structure than the mammalian cochlea, and the barn owl produces lots and lots of spontaneous emissions. The frequency map of this animal is extremely distorted, so if you were looking for localized sources due to pure anatomical irregularities, you might expect a distorted distribution of gaps between emissions. But in fact, the gaps remain stable throughout the entire frequency map, and the gaps between the emissions in terms of frequency represent exactly one cycle difference in the SFOAE.

John Guinan: I'm responding to the comment as to whether one should look for other kinds of propagation modes. Actually that's what the first part of my talk was about. The idea of finding emissions that were too short to be explained by the expected traveling wave peak in the apex, and the short group delay response derived from auditory nerve data (ANIP), I think of as arising from another propagation mode in the apex. I'm suggesting that [this apical mode] is what's giving rise to these shorter latency stimulus frequency emission components that we see in the apex of the guinea pig and maybe other animals as well. That's wasn't the modes you were talking about. But it is another kind of propagation mode.

Eric Le Page: One question we should ask relates to aging: What are the differential effects in terms of reflection, for example, due to a scattered hair cell loss. Second question is: Are we anywhere near the stage of going back to consider the original work on two [cochlear] turns, pre-1970, when this work was done purely psychophysically, and people talked in terms of consonance and dissonance, entrainment and roughness. For example, is the Knight and Kemp distinction [1999, 2000], the best way of describing the difference between consonance and roughness?

\section{Otoacoustic Emissions and Hearing Loss}

Yi-Wen Liu: I'm wondering [to which extent otacoustic emissions can be used as an] objective way to measure how badly hearing impaired people hear. In particular, can we estimate cochlear tuning using the DPOAE, because often when hearing is lost, the DPOAE is also gone.

John Guinan: Cochlear mechanics has a lot to tell us, but is not going to give a broad answer to how badly a hearing impaired person hears. It will just tell us part of it. My own hearing loss is not too bad at low frequencies, and very sharp at high frequencies. I'm sure I've also lost a lot of auditory nerve fibers, and that's not so much the mechanical problem.

Dáibhid Ó Maoiléidigh: Some evidence has been presented in mutants and in people with hydrops, where emissions have increased, for example, spontaneous emissions both in number and magnitude. And then, yes, in the population of people who show emissions, there has been a correlation between number of emissions and magnitude, and lower hearing thresholds and sharper tuning. However, in these mutants and in the hydrops case, the opposite is seen. Is there any framework where you can understand what's going on in the pathological cases and understand what we think is the paradigm for good tuning? We're often attempting to use a non-subjective measure of tuning by looking at distortion products, but yet there seem to be some effects that are contradictory to this.

Carolina Abdala: They're great questions. With my colleague, Radha Kalluri, we're thinking about how we can use OAEs more richly to describe hearing loss, versus simply categorize people as being hearing impaired or not. Based on the dual source model, perhaps we need to start thinking about extracting information from a reflection source emission and a nonlinear distortion emission that might tell us about the differences among types of sensory neural hearing loss. We know that those emissions are sensitive to different cochlear properties, but we don't know if we can use that to describe central neural hearing loss. Not impairments that have more neural involvement, but mild hearing loss, which by the way, outnumbers cases of deafness, and which produce notable handicaps in children. We devised one strategy for trying to exploit the two emission sources to characterize hearing loss and see if similar-deficit hearing losses might cluster in terms of these features. There is 
evidence that suggests (work I've done with Sumitrajit Dhar) that these two sources don't behave the same when you impose experimental manipulations, or even in natural aging and maturation, suggesting they are independent. Evidence from a mutant mouse (Mary Ann's [Cheatham] data were beautiful) along with the stereocilin deficient mouse, and the tectA and tectB, suggest that these emission sources can [indeed] vary independently. So why don't we start using [the richness of otoacoustic emissions] to probe the root of hearing loss? I'm hoping many more labs are interested in that question, because it's been 35 years since they were reported by David [Kemp], but we're still using a binary distinction of hearing loss or not, for most of the OAE applications.

\section{David Kemp: Thirty-six.}

Carolina Abdala: Thirty-six years. I was corrected.

Glenis Long: I want to go back to some of the early data. When we first started talking about spontaneous emissions, we talked about a group that was associated with normal hearing, and another group, usually larger, associated with damaged hearing. One prime example was our chinchilla at the Central Institute for the Deaf in St. Louis that had no spontaneous emissions until we noise exposed it. After exposure, SOAEs were located on either side of a discrete lesion along the basilar membrane. There's a family that Pat Wilson reported on, that had a lot of high frequency, large spontaneous emissions and had a dramatic hearing loss around those frequencies. But it's not inconsistent, with the models that we've been presenting, because remember, we're talking about reflection type emissions coming from some sort of irregularity. We're saying for the normal ones, we don't need a major irregularity, but if you have a major irregularity, then you will have large reflections which can give you some sort of spontaneous emissions.

Eric Le Page: Thanks Carolina [Abdala] for that comment. A couple of us here have been interested in the notion of using otoacoustic emissions as a greater measure of hearing loss. Back in 1998, I published a suggestion from transients, because transients are much more messy in terms of dividing it up into discrete mechanisms. But we did see a distinct decline in emission strength before there was any sign of hearing loss. I think this work was taken up by Lynne Marshall, who published some sort of confirmation of that.

Tatjana Tchumatchenko: I would like to comment on the applications versus understanding the problem. I think it's very helpful to have first a very thorough understanding before any application can take place, and this is something that the Max Planck Society is based on. In this sense, it will be also helpful to have multiple competing theories and multiple alternative ways to generate the same otoacoustic emission, and then test which of these alternative theories is useful in the application sense, rather than focusing on one or the other and then try to advance in one direction towards the application, so kind of try to open up the field more to alternative explanations for similar phenomena.

Paul Teal: Just a very quick question, which Chris [Shera] may be able to answer in relation to the stability of spontaneous otoacoustic emissions. Is there any evidence for that stability in surviving changes, particularly hearing loss, is associated with outer hair cells? Conductive hearing loss might change the middle ear impedance, while sensorineural hearing loss may change the irregularities.

Christopher Shera: Well, my impression is, as Glenis [Long] alluded to, that discrete lesions can induce spontaneous emissions, but generally speaking, spontaneous emissions, and actually reflection source emissions more generally, are pretty sensitive indicators of hearing loss and tend to be one of the first things that disappear.

Sumitrajit Dhar: My student, Rachel Baiduc was looking for spontaneous emissions at high frequencies that have typically been associated with lesions (published in Baiduc, Lee and Dhar, JASA 2014). She got tired of screening subjects, after about 120, she had found two spontaneous emissions that occurred along with reversals in threshold microstructure. But even at high frequencies, at least in the 120 people we screened, the majority of high frequency emissions seemed to behave just like regular spontaneous emissions.

Jonathan Siegel: One of the things I didn't have time to emphasize in our presentation leads into an interesting dichotomy. On one hand, as Mary Ann [Cheatham] presented, ears that seem to be close to generating their own spontaneous emissions or are, have unusually large stimulus frequency and transient emissions. That [observation] seems to be very sensitive to something that doesn't even seem to show up in behavioral thresholds or physiologically measured thresholds. So on the one hand, this sort of emission does seem to be very sensitive to small changes, but the flip side is, in Karolina [Charaziak] and my work, I showed 40 $\mathrm{dB}$ threshold shifts induced by tones that produced a much smaller decibel change in the stimulus frequency emission. And we found in separate measurements that using low-level two-tone stimulated distortion products can map the compound action potential, the threshold shifts virtually one $\mathrm{dB}$ per $\mathrm{dB}$. So in terms of actually measuring a correlate of permanent threshold shifts, the reflection source emissions don't seem to be particularly good.

Carolina Abdala: I know, your data puzzle me, but it's a chinchilla, and species-specific kind of patterns may exist, because the large scale studies Eric [Le Page] was talking about (also Michael Gorga and colleagues have done many, also Miller, et al, 2004) have routinely found that click evoked emissions, a reflection source emission, do in fact have sensitivities and predictive value for mild and slight hearing loss. We'd like to explore this because the idea is that these [emission types] might be more sensitive to those mild losses that tend to be missed in neonatal screening programs. At this point we accept the fact that we're going to 
miss mild hearing losses; perhaps we don't have to accept that, and that's why this research might be translational. One last comment about knowing the theory and generation before moving on. While I wouldn't suggest we truncate any kind of theoretical exploration, I think it has to be done concurrently. If you have a predictive value in a response you don't yet understand (exactly because it's very complex), I'm going to exploit that predictive value, even if there's still exploration going on.

Matt Flax: Consider the hearing issue neuropathy, where people can hear but actually can't comprehend because of certain neural problems. I believe there will be cases where you measure enhanced otoacoustic emissions, while there are actually hearing issues at those [cochlear frequency] locations [where the emission was generated], as well as the traditional concept of disappearing emissions. So we have to be prepared for the unexpected in that respect.

Dáibhid Ó Maoiléidigh: There was a paper from the Nuttall group, showing a spontaneous emission on the basilar membrane after damage, and the animal had increased [hearing] thresholds, and the emission was then correlated with spontaneous emissions. So at least in that case, correlates with some of the psychophysical evidence that damage can either increase or reduce emissions, and that it may sometimes be distinctive from a lower threshold. There's something there that maybe connects the two. If Fred [Nuttall] has a comment, it would be great.

Alfred Nuttall: Yes, we had only one guinea pig in many, many years of studying that had a spontaneous emission in the cochlear microphonic and on the basilar membrane. There are guinea pigs that have low frequency spontaneous cochlear microphonics around $1 \mathrm{kHz}$. They are more frequent and I have read (I couldn't give you the citation), that if not anesthetized, guinea pigs have more of these low frequency spontaneous oscillations. I don't know if they appear as otoacoustic emissions. However, our $15 \mathrm{kHz}$ emission was not only present as cochlear microphonic, but it was very loud as an acoustic emission, and that's how we recognized it. We could hear it and realized that there was something strange about this experiment. We had a noise in our sound isolated booth, and that clued us in that something was going on. And we had all kinds of funny things happening, for example. When we opened the booth door to investigate this, we had the emission entrained by sounds coming from outside the room. We had $17 \mathrm{kHz}$ fly back frequencies on our oscilloscope. And that sound was going into the room, and interfering with the emission. So we had a lot of fun with that animal and got as much data as we could out of it, and then published that data.

Sumitrajit Dhar: I'd like to comment on the discussion about whether theory should trail practice or not. I'd like to submit to this group that our practice is disgracefully trailing theory today. One example of that is the discussion here about the use of otoacoustic emissions for human hearing loss. All hearing loss is not the same, and we happen to know something about how these are generated. So why not target types of hearing loss that are more likely to be affected by outer hair cell damage and forget about hearing loss in general?

Sarah Verhulst: So with that said, I think we shall continue our discussions over lunch, and I thank you all for your participation. 